\title{
Analysis and expression of Pmlyzi3 from Penaeus monodon
}

\author{
Chaogang Wang ${ }^{1,2}$, Guoqiang $\mathrm{Li}^{1,2}$, Liang Zhou ${ }^{3}$, Anguo Li ${ }^{1,2}$, Chenjing Shang ${ }^{1,2}, \mathrm{Xu}$ Deng ${ }^{1,2, *}$ \\ ${ }^{1}$ Shenzhen Key Laboratory of Marine Bioresource \& Eco-environmental Science, College of Life Sciences and Oceanography, Shenzhen \\ University, Shenzhen 518060, PR China \\ ${ }^{2}$ Guangdong Technology Research Center for Marine Algal Bioengineering, College of Life Sciences and Oceanography, Shenzhen \\ University, Shenzhen 518060, PR China \\ ${ }^{3}$ Shenzhen Institute of Synthetic Biology, Shenzhen Institutes of Advanced Technology, Chinese Academy of Sciences, Shenzhen, China
}

\begin{abstract}
Lysozymes are crucial immune moleculars and play an important role in innate imunity. Here, a new lysozyme named Pmlyzi3 was found from the transcriptome data of Panaeus monodon. The Pmplyzi3 gene was $438 \mathrm{bp}$ in length, encoding a 146-residues peptide and the first 19 residues constituted a signal peptide. The mature peptide contained 10 cysteines and had $7 \alpha$-helixes in its $\mathrm{N}$ terminal. Moreover, it showed $88 \%$ identity with lysozyme-like protein from Penaeus vannamei. To express Pmlyzi3, pColdIV-SUMOPmlyzi3 plasmid was constructed by linked the Pmlyzi3 with SUMO tag, then transformed to Eschericha coli BL21 (DE3). By optimizing expression condition, SUMO-Pmlyzi3 was succeeded in expression in high level and purifing with Ni-NTA column. Following with SUMO protease excision, pure Pmlyzi3 was obtained by removing SUMO tag, which would be helped to study its function.
\end{abstract}

\section{Introduction}

Antimicrobial peptides (AMPs), as an impoortant immune molecular, owns indispensible function in innate imunity[1]. AMPs normally exist in many kinds of organisms against variety of bacteria, fungi, parasite, virus and cancer cells[2], so that researchers think that it would be the best canditate for antibiotic. Comparing with tranditional antibiotics, AMPs is not easy to cause bacterial resisitance[3]. Till now, over 2960 AMPs were found and 2184 of them were from animals[4].

Invertebrates depend on innate immunity to defend invading microbes because they lacked adaptive immunity. It is well known that immunity was composed of cellular immunity and humoral imumuity, and AMPs belonged to the humoral immnity system[5]. Several types of AMPs were found in crustaceans, including antilipopolysaccharide factors (ALFs), penaeidins, crustins and lysozymes[6,7]. Penaeidins, crustins and ALFs were reported in recent years $[8,9]$.

Lysoozyme, as a cationic protein, normally existed in animal, plant and microorganism. They killed microbes by destroyed the $\beta-1,4$-glycoside bond which made the cell wall hydrolyze and the contents leak out. Gernally, there were three types of lysozymes, including chickentype lysozyme (c-type), goose-type lysozyme (g-type) and invertebrate-type lysozyme (i-type)[10]. The c-type was found in viruses, bacteria, plants, insects, reptilles, poultry, fish and mammals[11], g-type existed in birds, fish and scallop[13], while i-type normally was in invertebrate. There were over 20 i-type lysozymes including the first found in Asterias rubens [13-15].
Lysozymes were normally involved in defensive mechanisms including lysis, immune response and antimicrobial and antiviral activities [16]. Recombinat expression of c-type lysozyme from Litopenaeus vannamei showed notable antimicrobial activities to vibro alginolyticus and vibrio cholerae [17]. The c-type and itype lysozymes from Penaeus japonicus and Penaeus monodon also owned antimicrobial activities to gramnegative and gram-positive bacteria [10][18].

Though c-type lysozyme from Penaeus japonicus and Penaeus monodon were reported in many articles, i-type lysozyme had few reports. Hence, we obtained a new itype lysozyme from transcriptome data of Penaeus monodon. Recombinant expression of this lysozyme in Escherichia coli and analysis of its antimicrobial activity were proceed and significant results were obtained.

\section{Materials and Methods}

\subsection{Materials}

\subsubsection{Vector and strain}

pColdIV vector was bought from General Biology (Anhui) System Co., Ltd and competent cell of E.coli BL21 (DE3) was from Shanghai Local Biotechnology Co., Ltd.

\subsection{Bioinformatics analysis of Pmlyzi3}

The nucleotide sequence of Pmlyzi3 was obtained from transcriptome sequencing data of the hepatopancreas of

\footnotetext{
* Corresponding author: dengxu@szu.edu.cn
} 
Penaeus monodon (unpublished data). The Open Reading Frame and amino acid sequence of Pmlyzi3 were deduced by ORF Finder (http://www.ncbi.nlm.nih.gov/gorf/gorf.html). The signal peptide was predicted with signalP 4.0 server. DNAman version 6 was used for generating gene structure information. Homologous sequences of Pmlyzi3 were obtained by Basic Local Alignment Search Tool (BLASTP) and multisequences alignment was performed with MEGA X. The physicochemical properties were predicted with the online software Protparam (http://web.expasy.org/protparam/).

\subsection{Gene synthesis and vector construction}

The mature peptide of Pmlyzi3 was fused to the Cterminus of a SUMO protein with His-tag and overexpressed in Escherichia coli BL21 (DE3). The nucleotide sequence of His-SUMO-Pmlyzi3 with NdeI and $\mathrm{SacI}$ cut sites in both ends was codon optimized for $E$. coli and chemically synthesized (General Biosystems, Inc., Hefei, China). The synthesized DNA was then linked to pColdIV vector by NdeI and SacI cut sites. The reconstituted plasmid was transformed to E. coli BL21 Rosetta (DE3), named pColdIV-SUMO-Pmlyzi3.

\subsection{Verification of recombinant plasmid}

Recombinant plasmid verified through PCR with primers of pColdIV-F and pColdIV-R. pColdIV-F was as : 5'ACGCCATATCGCCGAAAGG-3' and pColdIV-R was as 5'-GGCAGGGATCTTAGATTCTG-3'. The PCR was performed with $94{ }^{\circ} \mathrm{C}, 3 \mathrm{~min} ; 40$ cycles of $94{ }^{\circ} \mathrm{C}, 20 \mathrm{~s}$ and $58^{\circ} \mathrm{C}, 20 \mathrm{~s} ; 72^{\circ} \mathrm{C}, 5 \mathrm{~min}$. The PCR product was checked by electrophoresis on a $1 \%$ agarose gel.

\subsection{Protein expression in E.coli}

The recombinant plasmid was transformed to E.coli BL21 Rosetta (DE). Adding $20 \mathrm{~mL}$ of overnight cultivated transgenic E.coli into the fresh $2 \mathrm{~L} \mathrm{LB}$ broth medium containing ampicillin $(50 \mu \mathrm{g} / \mathrm{mL})$ and then cultivating them at $37{ }^{\circ} \mathrm{C}$ with shaking at $200 \mathrm{rpm}$ until the absorbance reached 0.5 at $600 \mathrm{~nm}$. IPTG was added to the culture at a final concentration of $1 \mathrm{mM}$. The induction was performed at $16^{\circ} \mathrm{C}$ for 6 hours. Cells were harvested by centrifugation and the cell pellets were washed and resuspended in PBS. The bacterial suspensions were then disrupted by ultrasonication for 40 min (power: 195W, open for 2 seconds, stop for 4 seconds). The supernatant (soluble fraction) was collected and analyzed by SDSPAGE.

\subsection{Optimization the expression of SUMO- Pmlyzi3}

When induction with IPTG, culture was sampled after induced $0,1.5,3,4.5,6,7.5$ and $9 \mathrm{~h}$. The IPTG concentration was chosen at $0.2,0.4,0.6,0.8 \mathrm{mM}$. All the samples were checked by SDS-PAGE.

\subsection{Protein purification}

The fusion protein was purified by Ni-NTA Sepharose Fast Flow (Ruidahenghui, Beijing, China), and the histagged protein was eluted with buffer contained $50 \mathrm{mM}$ PBS, $300 \mathrm{mM} \mathrm{NaCl}$ and $200 \mathrm{mM}$ imidazole. Elutes were further analyzed by SDS-PAGE.

The eluted protein was further dialyzed against $50 \mathrm{mM}$ Tris- $\mathrm{HCl}, 200 \mathrm{mM} \mathrm{NaCl}$ to remove the imidazole and then quantified by Bradford reagent (Sangon, China). 100 $\mu \mathrm{g}$ protein was mixed with $1 \mathrm{U}$ SUMO protease (General Biosystems, Inc., Hefei, China), and incubated at $4^{\circ} \mathrm{C}$ overnight to remove the SUMO tag. The pure Pmlyzi3 was treated with $50 \%$ acetonitrile, then $100 \%$ acetonitrile to remove coomassie brilliant blue. The supernatant was discarded and the gel was treated with pancreatin $(25 \mu \mathrm{g} / \mathrm{mL})$, then extract solution (acetonitrile and $\mathrm{H}_{2} \mathrm{O}$ in $1: 4$, followed by $0.5 \%$ formic acid in total volume). The powder was obtained by ultrasonography and vacuum drying, and then dissolved in LC-MS buffer, finally identified by LC-MS.

\section{Results}

\subsection{Identification of Pmlyzi3}

Pmlyzi3 was obtained from the transcriptome after blast. The Pmplyzi3 gene was confirmed by RT-PCR and it was 438 bp in length, encoding a 146-residues peptide and the first 19 residues constituted a signal peptide (Figure 1). The 127-residues mature peptide contained 10 cysteines and had $7 \alpha$-helixes in its $\mathrm{N}$ terminal (Figure 2). The molecular weight was $14.279 \mathrm{kDa}$ and the theoretical $\mathrm{p} I$ was 4.62 .

\subsection{Alignment of Pmlyzi3 with other lysozymes}

Pmlyzi3 showed $88 \%$ identity with lysozyme-like from Penaeus vannamei, while $60 \%$ with lysozyme from Eriocheir sinensis. However, Pmlyzi3 had low identity with other lysozyme, even Penmonlys-like type from Penaeus monodon (Figure 3).

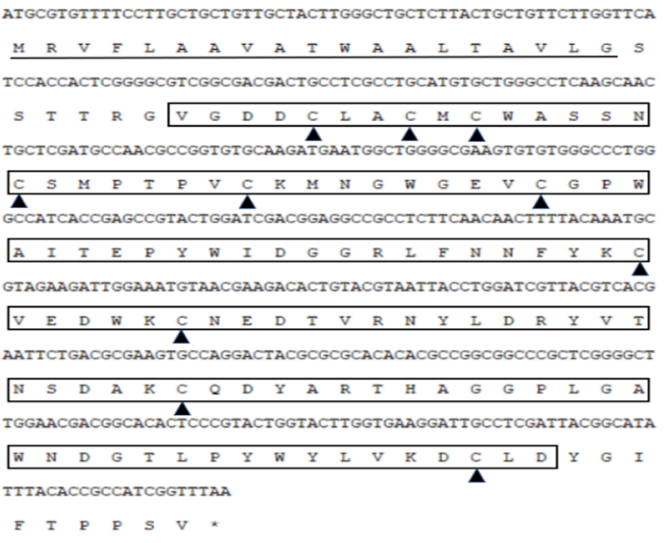

Fig. 1.Nucleotide and amino acid sequence of Pmlyzi3. The signal peptide was underlined; the cysteines were labeled in black triangle. 


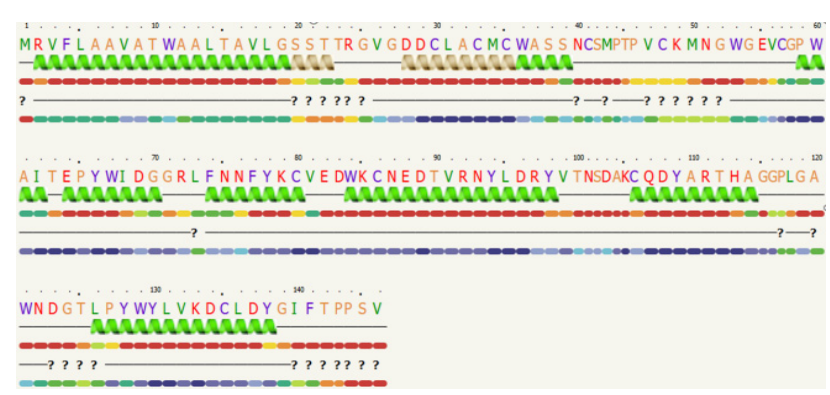

Fig. 2. Protein secondary structure of Pmlyzi3. $\alpha$-helix was in green.

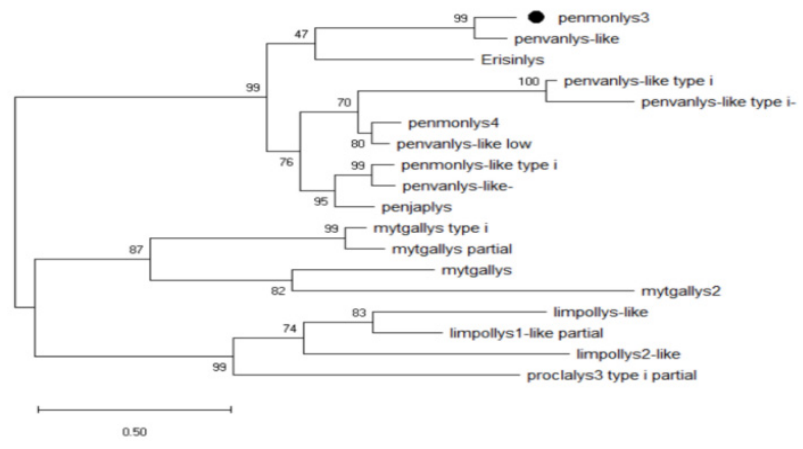

Fig.3. Molecular phylogenetic tree of Pmlyzi3 from Penaeus monodon.

The GenBank IDs of lysozymes sorted by species are as follows: Penaeus vannamei (Penvanlys-like: XP_027231470.1; Penvanlys-like-: XP_027212812.1; Penvanlys-like low: XP_027212212.1; Penvanlys-like type i-1: ROT76628.1; Penvanlys-like type i-1-: ROT76627.1) ; Penaeus japonicus (Penjaplys: AMO44057.1) ; Penaeus monodon (Penmonlys-like type I: ACZ63471.1) ; Eriocheir sinensis (Erisinlys: AEU04535.1); Mytilus galloprovincialis (Mytgallys type I: AJQ21515.1; Mytgallys-partial: OPL33781.1; Mytgallys: AAN16210.1; Mytgallys2: BAF63423.1) ; Procambarus clarkia (Proclalys3 type I: AVK42824.1) ; Limulus polyphemus (Limpollys-1: XP_022236369.1; Limpollys1-1 partial: XP_022236677.1; Limpollys2-1: XP_013792023.1);

\subsection{Vector construction, recombinant expression of Pmlyzi3}

The mature peptide of Pmlyzi3 was linked with SUMO protein with His- tag, then ligated to pColdIV with NdeI and SacI. PCR results showed that SUMO-Pmlyzi3 could be amplified from pColdIV-SUMO-Pmlyzi3 with primers of pColdIV-F and pColdIV-R (Figure 4).

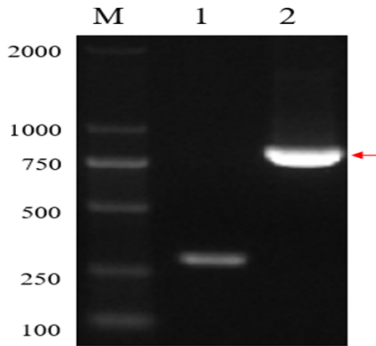

Fig.4. Verification of recombinant plasmid M: DL 2000 marker; 1: negative control; 2: pColdIV-SUMO-Pmlyzi3

\subsection{Expression of SUMO-Pmlyzi3}

pColdIV-SUMO-Pmlyzi3 was transformed to E.coli BL21 (DE3) and then transformants were induced by $1.0 \mathrm{mM}$ IPTG for $6 \mathrm{~h}$, showing that SUMO-Pmlyzi3 was expressed with the weight of $25 \mathrm{kDa}$ and most of the recombination protein was soluble protein (Figure 5).

According to our results, the best protein expression condition was induced with $0.8 \mathrm{mM}$ IPTG for $4.5 \mathrm{~h}$.

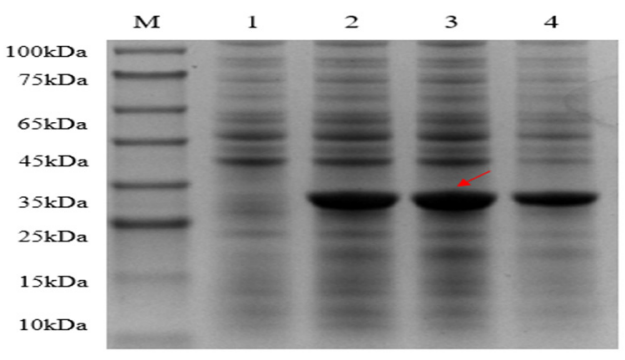

Fig.5. Expression of SUMO-Pmlyzi3

M: protein marker; 1: without induction; 2: induction with 1.0mM IPTG for $6 \mathrm{~h} ; 3$ : supernatant after cell disruption; 4 : sediment after cell disruption

\subsection{Purification of Pmlyzi3}

The His-SUMO-Pmlyzi3 fusion protein was expressed in E.coli induce with IPTG and then purified with a Ni-NTA column, finally eluted by $500 \mathrm{mM}$ imidozol as a pure protein (Figure 6).

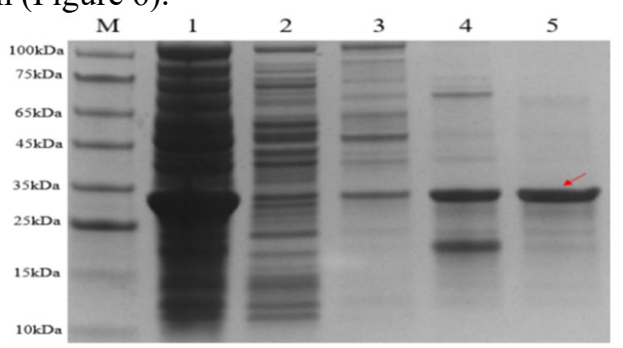

Fig.6. Purification of SUMO-Pmlyzi3 by Ni-NTA column M: protein marker; 1:sample, which is the supernatant after cell disruption; 2: flowthrough; 3 : wash off protein with $100 \mathrm{mM}$ imidazole; 4, 5: eluted proteins with $200 \mathrm{mM}$ and $500 \mathrm{mM}$ imidazole

\subsection{Obtaining Tag-free Pmlyzi3}

After incubated with 0.5U SUMO protease, 50 $\mu \mathrm{g}$ SUMO- 
Pmlyzi3 was succeeded in removing SUMO tag (Figure 7). Following with LC-MS analysis, tag-free $P m$ lyzi3 was confirmed that there was no other residues except that of Pmlyzi3 (Figure 8).

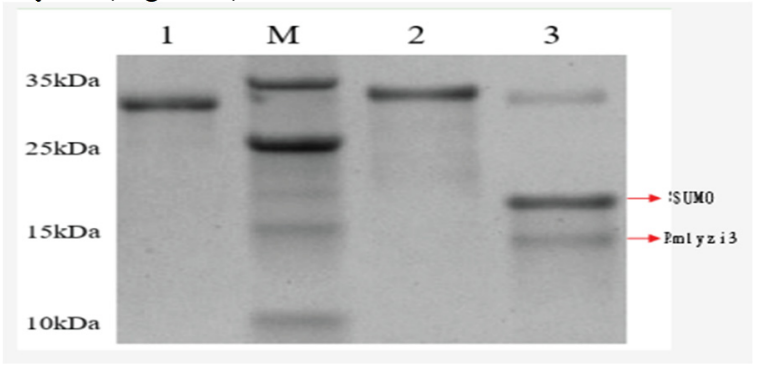

Fig.7. Cleavage of SUMO tag

M: protein marker; 1: SUMO-Pmlyzi3; 2: SUMO protease; 3: protein after cleavage, with SUMO and Pmlyzi3 indicated

\section{Protein sequence coverage: $\mathbf{2 2} \%$}

Matched peptides shown in bold red.

1 SSTTRGVGD CLACMCWASS NCSMPTPVCK MNGWGEVCGP WAITEPYWID 51 GGRLFNNEYK CVEDWKCNED TVRNYLDRYV TNSDAKCQDY ARTHAGGPLG 101 AWNDGTLPYW YLVKDCLDYG IFTPPSV

Fig.8. LC-MS analysis of the enzyme digested product of SUMO-Pmlyzi3

\section{Discussion}

A new lysozyme named Pmlyzi3 was found from the transcriptome data of $P$. monodon after bioinformatics analysis. Most of the lysozymes found in shrimp were ctype or i-type[19]. They owned a LYZ1 domain composed of 10 cysteines[20].

Antimicrobial peptide was hard to achieve high level expression because its molecular weight was low and they existed anti-bacterial action. The present results showed that SUMO as the molecular chaperone was helped to express antimicrobial peptide[21]. Hence, we employed SUMO as molecular chaperone to express antimicrobial peptide in E.coli. Considering that antimicrobial peptide was easy to form inclusion, SUMO-Pmlyzi3 was induced in low temperature. Due to the his-tag, we can purify recombinant protein by Ni-NTA column[22]. After treated with SUMO protease, Pmlyzi3 was freed from fusion protein so that we obtained pure Pmlyzi3, which would be helped to study its function.

\section{Acknowledgements}

This research was supported by the National Natural Science Foundation of China [31470389, 41706137], the Natural Science Foundation of Guangdong Province (2017A030310332), the Shenzhen Grant Plan for Science \& Technology [JCYJ20160422171614147, JCYJ20170818101523761], the Natural Science Foundation of SZU (2019078), State Key Laboratory of Tropical Oceanography, South China Sea Institute of Oceanology, Chinese Academy of Sciences (Project No. LTO1909).

\section{References}

1. R E.W. Hancock, K.L. Brown, and N. Mookherjee, Immunobiology. 211, 4 (2006).

2. L. Zhang, R L. Gallo, Curr Biol. 26, 1 (2016).

3. P. Bulet, R. Stöcklin, L. Menin, Immunol Rev. 198, 1 (2010).

4. H. Moravej, Z. Moravej, M. Yazdanparast, and M. Heiat, Microb Drug Resist. 24, 6 (2018).

5. A. Tassanakajon, V. Rimphanitchayakit, S. Visetnan, P Amparyup, K. Somboonwiwat, W. Charoensapsri, and S. Tang, Dev Comp Immunol. 80 (2018).

6. A. Tassanakajon, K. Somboonwiwat, P. Supungul, and S. Tang, Fish Shellfish Immun. 34, 4 (2013).

7. D. Destoumieux-Garzón, R.D. Rosa, P. Schmitt, C. Barreto, J. Vidal-Dupiol, G. Mitta, Y. Gueguen, and E. Bachère, Philos T R Soc B. 371, 1695 (2016)

8. P.S. Gross, T.C. Bartlett , C.L. Browdy, R.W. Chapman, G.W. Warr, Dev Comp Immunol. 25, 7 (2001).

9. P. Supungul, S. Klinbunga, R, Pichyangkura, S. Jitrapakdee, I. Hirono, A. Tassanakajon, Mar Biotechnol. 4, 5 (2002).

10. P. Supungul, V. Rimphanitchayakit, T. Aoki, I. Hirono, and A Tassanakajon, Fish Shellfish Immun. 28, 3 (2010).

11. W. Mai, C. Hu, Mol Biol Rep. 36, 6 (2009).

12. J. Zhao, L. Song, C. Li, H. Zou, D. Ni, W. Wang, and W. Xu, Mol Immunol. 44, 6 (2007).

13. Q. Ren, Y.L. Qi, K.M. Hui, Z. Zhang, C.Y. Zhang, and W. Wang, Fish Shellfish Immun. 33, 4 (2012).

14. S. Bachali, M. Jager, A. Hassanin, F. Schoentgen, P. Jollès, A. Fiala-Medioni, and J.S. Deutsch, J Mol Evol. 54, 5 (2002).

15. J. Zhao, L. Qiu, X. Ning, A. Chen, H. Wu, and C. Li, Comp Biochem Physiol B Biochem Mol Biol. 156, 1 (2010).

16. W. Mai, W. Wang, Fish Shellfish Immun. 28, 4 (2010).

17. E. De-La-Re-Vega, A. García-Galaz, M.E. DíazCinco, and R.R. Sotelo-Mundo, Fish Shellfish immune. 20, 3 (2006).

18. S. Hikima, J. Hikima, J. Rojtinnakorn, I. Hirono, and T. Aoki, Gene. 316 (2003).

19. L.L. Shi, T.T. Zhou, Y.Q. Li, and K.H. Zeng, Journal of Guangdong Ocean University. 37, 3 (2017).

20. Z.Q. Du, and T. Lin, Hubei Agricultural Sciences. 52, $22(2013)$.

21. Y. Li, Appl Biochem Biotech. 54, 1 (2009).

22. X.M. Yang, J.Z. He, L. Zhang, X.J. Huang, Y.F. Guo, and H.S. Jiang, Biotechnology Bulletin. 10 (2010). 